\title{
Optimal Strategy for QoS Provision under Spectrum Mobility in Cognitive Radio Networks
}

\author{
Tao Guo and Klaus Moessner \\ Centre for Communication Systems Research, University of Surrey \\ Guildford, GU2 7XH, United Kingdom \\ Email: \{t.guo, k.moessner\}@surrey.ac.uk
}

\begin{abstract}
In cognitive radio networks, the arrival of Primary Users (PUs) may force Secondary Users (SUs) to terminate their ongoing sessions or degrade their Quality of Service (QoS) level. Given the time-varying spectrum availability, an immediate challenge arising is to support the QoS of SUs under spectrum mobility. In this paper, we propose an optimal decision-making framework for joint admission control, eviction control and bandwidth adaptation in cognitive radio networks. The problem is formulated as a Semi-Markov Decision Process (SMDP) and the optimal decision for each system state is derived to maximize the long-term network revenue as a function of the spectrum utilization, the SU blocking probability and the bandwidth adaptation cost under the SU dropping probability constraint. It is shown that the derived optimal strategy outperforms the threshold-based channel reservation schemes with/without bandwidth adaptation. And among the schemes with bandwidth adaptation, more performance improvement can be achieved by the proposed one when the bandwidth adaptation cost is taken into account.
\end{abstract}

\section{INTRODUCTION}

Static spectrum allocation policy currently regulated by governmental agencies has led to an inefficient utilization of the precious spectrum resources. While some portions of the spectrum are becoming more and more crowded such as the GSM and $3 \mathrm{G}$ bands, other portions of the spectrum are underutilized such as the broadcast TV bands. Cognitive radio [1] has been recognized as a promising technology to exploit the underutilized spectrum resources. It allows unlicensed Secondary Users (SUs) to opportunistically access the unused spectrum of the licensed Primary Users (PUs). However, the PUs have the priority over the SUs and their activities should not be affected by the SUs. When a frequency band is reclaimed by a PU, the SUs on this band should be handed over to other idle bands or evicted in case that no other idle bands are available. Given the time-varying nature of the licensed spectrum availability, an immediate challenge arising is to support the Quality of Service (QoS) of the SUs under spectrum mobility.

Recent work [2] proposes to reserve a certain number of channels as backup channels for potential spectrum handover to reduce the forced dropping probability of the SUs. The optimal number of reserved channels has been found by modelling the process of spectrum occupation as a continuoustime Markov chain. [3] investigates admission control schemes with fractional guard channel, in which a non-integer number of channels can be reserved for spectrum handover. The above threshold-based channel reservation schemes cannot fully exploit the statistic availability of the spectrum and are difficult to provide bounded limit for the forced dropping probability of the SUs. [4] proposes an adaptive call admission control scheme with soft-QoS based spectrum handover. However, it is specified that a new SU will be accepted only when there are enough channels to support its full rate service. In addition, the minimum dropping probability optimization is formulated as a nonlinear programming problem solved via a relaxation method. Therefore, their proposed approach cannot lead to an optimal solution for fully exploiting the adaptive admission control and QoS control.

From a user's perspective, a block of a new session or a complete termination of an ongoing session is more annoying than compromising the QoS to certain extent but still initiating or maintaining the session at an accepted QoS level. In practice, various technologies have been adopted to adaptively degrade and upgrade the QoS level of a session depending on the varying resource availability. For example, layered encoding schemes have been used for multimedia applications [5]. Depending on the bandwidth availability, different encoding schemes can be adaptively used to keep the session continuity. In a cognitive radio network, bandwidth adaptation mechanism could be especially useful. When few PUs are present, more spectrum bands can be allocated to the SUs to upgrade their QoS levels. When more PUs appear, the SUs may degrade their QoS levels by giving up some spectrum bands but no user is evicted as long as the minimum QoS requirement can be satisfied. In this paper, we investigate the joint admission control, eviction control and bandwidth adaptation problem in cognitive radio networks. In particular, we formulate the problem as a Semi-Markov Decision Process (SMDP) to derive the optimal decisions for each system state during the lifetime of the system. The objective is to maximize the long-term network revenue as a function of the spectrum utilization, the SU blocking probability and the bandwidth adaptation cost while keeping the forced dropping probability of the SUs upper-bounded. An optimal solution has been developed in [6] to decide whether or not to admit and evict a $\mathrm{SU}$ of a specific bandwidth requirement. However, bandwidth adaptation is not considered in their work, and thus, spectrum utilization is not fully exploited whereas SUs will encounter high blocking and dropping rate under spectrum mobility. Call admission control schemes for adaptive multimedia have been 
an active research area in conventional cellular networks [7] [8]. However, the solutions there cannot be simply borrowed into cognitive radio networks due to the absence of the roles of PUs and SUs.

The rest of the paper is organized as follows. Section II describes the system model. Section III formulates the joint admission control, eviction control and bandwidth adaptation problem as a SMDP. Section IV presents a Linear Programming (LP) based method to obtain the optimal solution of the SMDP. The performance of the proposed scheme is evaluated in Section V. Finally, Section VI summarizes the key findings and draws a conclusion.

\section{System ModeL}

It is assumed that the licensed spectrum consists of $M$ primary frequency bands and the capacity (or bandwidth) of each primary band is $C$. The total bandwidth of $M \times C$ are shared by PUs and SUs. The PUs has the priority over the SUs on the utilization of the spectrum bands and the SUs must vacate the bands when they are reclaimed by the PUs. A PU will use one primary band for transmission whereas a SU can use one of the bandwidths among $\left\{B_{1}, B_{2}, \ldots, B_{K}\right\}$ for transmission, where $B_{\min }=B_{1}<B_{2}<\ldots<B_{K}=B_{\max }$, based on the spectrum availability. Ideally, a SU will prefer to use the maximum bandwidth for transmission for the best user's Quality of Experience (QoE). However, if this cannot be satisfied due to the lack of spectrum resource, the transmission can still continue as long as the minimum bandwidth requirement can be supported. We assume that Orthogonal Frequency Division Multiplexing (OFDM) technique is used for SU transmission, and thus, when a primary band is reclaimed by a PU, the SUs using any portion of this band can be easily migrated to other bands with idle bandwidths by remapping the OFDM subcarriers. We assume that the PU and the SU arrival processes follow a Poisson process with arrival rate $\lambda_{p}$ and $\lambda_{s}$, respectively. The service time of a PU and a SU follows an exponential distribution with mean $1 / \mu_{p}$ and $1 / \mu_{s}$, respectively. These assumptions have been widely used in the literature and shown to achieve a good balance between the real traffic characteristics and the mathematical tractability.

\section{SMDP FORMULATION}

The decision-making process for joint admission control, eviction control and bandwidth adaptation is modelled as a SMDP. SMDPs are widely used to model stochastic control problems in dynamic systems satisfying Markov property. At a decision epoch in a dynamic system, the system is in one of the states in a finite state space. An action is chosen from a finite action space and moves the system to a new state. A corresponding reward/cost is incurred due to the action taken. Markov property means that the time until, the new state at, and the reward/cost incurred until the next decision epoch depend only on the current state and the action taken. Given the assumptions made in Section II, the process considered in this paper possesses Markov property and can be modelled as a SMDP. The proof is omitted here due to page limit.

\section{A. System States}

At any time instant, the state of the system is represented as $\mathbf{s}=(m, \mathbf{n})$, where $m$ is the number of active PUs in the system, $\mathbf{n}=\left(n_{1}, n_{2}, \ldots, n_{K}\right)$ and $n_{i}(1<=i<=K)$ denotes the number of SUs using the bandwidth $B_{i}$ for transmission. Given the total capacity constraint of the spectrum, the state space is:

$$
\mathbf{S}=\left\{\mathbf{s}: 0 \leq m \leq M, \sum_{i=1}^{K} n_{i} \leq(M-m) C\right\} .
$$

\section{B. Actions}

Similar to [9] [7], a decision at a state is made before the occurrence of the next event, i.e. the decision maker should pre-decide the actions for all the possible events at the next decision epoch. At a decision epoch, one of the following four events can occur: (1) a SU arrival; (2) a PU arrival; (3) a SU departure; (4) a PU departure. Thus, an action at a decision epoch can be denoted as:

$$
\mathbf{a}=\left(a_{s}, a_{p}, e, \mathbf{b}_{\mathbf{s a}}, \mathbf{b}_{\mathbf{p a}}, \mathbf{b}_{\mathbf{s d}}, \mathbf{b}_{\mathbf{p d}}\right),
$$

where $a_{s}$ and $a_{p}$ stands for the admission control policy for the arrival of a SU and a PU respectively (admit $\Leftrightarrow a_{s}, a_{p}=1$, reject $\Leftrightarrow a_{s}, a_{p}=0$ ), $e$ denotes the number of SUs to be evicted when a PU arrives, $\mathbf{b}_{\mathrm{sa}}$ denotes the action of bandwidth allocation of the new SU and bandwidth reallocation of the existing SUs when a $\mathrm{SU}$ arrives, $\mathbf{b}_{\mathbf{p a}}$ denotes the action of SU eviction and bandwidth reallocation when a PU arrives, $\mathbf{b}_{\mathrm{sd}}$ and $\mathbf{b}_{\mathrm{pd}}$ denote the action of bandwidth reallocation when a SU departs and when a PU departs respectively.

$a_{s}=0$ is a possible action for every state and $a_{s}=0$ is the only possible action when all the spectrum bands have been occupied by the PUs. Thus, we have:

$$
a_{s}=\left\{a_{s} \in\{0,1\}: a_{s}=0 \text { if } m=M\right\} .
$$

Since the PUs have the priority over the SUs for spectrum access, the admission control policy for the PUs is deterministic:

$$
a_{p}=\left\{a_{p}=1 \text { if } m<=M-1, a_{p}=0 \text { if } m=M\right\} .
$$

The action space of $e$ is: $e \in\left[0, \sum_{i=0}^{K} n_{i}\right]$.

The form of $\mathbf{b}_{\mathbf{s a}}$ and its action space is defined as:

$$
\begin{aligned}
\mathbf{b}_{\mathbf{s a}} & =\left\{a_{s}\left(b_{s a}^{1}, b_{s a}^{2}, \ldots, b_{s a}^{K}\right): \sum_{i=1}^{K} b_{s a}^{i}=1,\right. \\
& -n_{i} \leq b_{s a}^{i} \leq\left\lfloor\frac{(M-m) C}{B_{i}}\right\rfloor-n_{i} \text { for } 1 \leq i \leq K, \\
& \sum_{i=1}^{K} B_{i}\left(n_{i}+b_{s a}^{i}\right) \leq(M-m) C, \\
& \text { if } \left.\exists j\left(1 \leq j \leq K, b_{s a}^{j}<0\right) \text { then } b_{s a}^{i} \leq 0 \text { for } \forall i>j\right\} .
\end{aligned}
$$

where $b_{s a}^{i}(1 \leq i \leq K)$ denotes the variation of the number of the SUs using the bandwidth $B_{i}$ when a new SU arrives. The first constraint indicates that if a SU is admitted the total number of SUs in the system should be increased by 1 . The second constraint gives the range of the SU number variation. 
The third one is the total system capacity constraint. In order to admit the new SU, some existing SUs in the system may need to give up some spectrum resources they are currently using. However, the vacated spectrum should not be used by other SUs to upgrade their QoS level. And the bandwidth allocated to the new SU should not exceed the bandwidth allocated to any existing SU. This requirement is enforced by the last constraint.

Similarly, the form of $\mathbf{b}_{\mathbf{p a}}$ and its action space is defined as:

$$
\begin{aligned}
\mathbf{b}_{\mathbf{p a}} & =\left\{a_{p}\left(b_{p a}^{1}, b_{p a}^{2}, \ldots, b_{p a}^{K}\right): \sum_{i=1}^{K} b_{p a}^{i}=-e,\right. \\
& -n_{i} \leq b_{p a}^{i} \leq\left\lfloor\frac{(M-m-1) C}{B_{i}}\right\rfloor-n_{i} \text { for } 1 \leq i \leq K, \\
& \sum_{i=1}^{K} B_{i}\left(n_{i}+b_{p a}^{i}\right) \leq(M-m-1) C, \\
& \text { if } \left.\exists j\left(1 \leq j \leq K, b_{p a}^{j}<0\right) \text { then } b_{p a}^{i} \leq 0 \text { for } \forall i>j\right\} .
\end{aligned}
$$

where $b_{p a}^{i}(1 \leq i \leq K)$ denotes the variation of the number of the SUs using the bandwidth $B_{i}$ when a new PU arrives. The first constraint indicates that the total number of the evicted SUs is equal to $e$. The rest constraints have the similar meaning as above.

The form of $\mathbf{b}_{\mathbf{p d}}$ and its action space is defined as:

$$
\begin{aligned}
\mathbf{b}_{\mathbf{p d}} & =\left\{\left(b_{p d}^{1}, b_{p d}^{2}, \ldots, b_{p d}^{K}\right): \sum_{i=1}^{K} b_{p d}^{i}=0,\right. \\
& -n_{i} \leq b_{p d}^{i} \leq\left\lfloor\frac{(M-m+1) C}{B_{i}}\right\rfloor-n_{i} \text { for } 1 \leq i \leq K, \\
& \sum_{i=1}^{K} B_{i}\left(n_{i}+b_{p d}^{i}\right) \leq(M-m+1) C, \\
& \text { if } \left.\exists j\left(1 \leq j \leq K, b_{p d}^{j}>0\right) \text { then } b_{p d}^{i} \geq 0 \text { for } \forall i>j\right\} .
\end{aligned}
$$

where $b_{p d}^{i}(1 \leq i \leq K)$ denotes the variation of the number of the SUs using the bandwidth $B_{i}$ when a PU departs. The first constraint indicates that the total number of the SUs in the system is unchanged. The second and third constraints are similar to the above. The last constraint limits that only the spectrum released by the departed PU should be reallocated among the SUs.

The operation of bandwidth reallocation upon a SU departure event depends on the amount of the spectrum released by the departed SU. Thus, $\mathbf{b}_{\mathbf{s d}}$ is further defined as $\mathbf{b}_{\text {sd }}=\left(\mathbf{b}_{\text {sd }}^{\mathbf{1}}, \mathbf{b}_{\mathbf{s d}}^{\mathbf{2}}, \ldots, \mathbf{b}_{\text {sd }}^{\mathbf{K}}\right)$, where $\mathbf{b}_{\text {sd }}^{\mathbf{k}}(1 \leq k \leq K)$ denotes the bandwidth reallocation operation when a SU using the bandwidth $B_{k}$ departs. Let $\mathbf{n}_{\mathbf{k}}^{\prime}=\left(n_{1, k}^{\prime}, n_{2, k}^{\prime}, \ldots, n_{K, k}^{\prime}\right)$ denote the new state of the number of SUs using different bandwidths after a SU using the bandwidth $B_{k}$ departs. We have $n_{i, k}^{\prime}=n_{i}-1$ for $i=k$ and $n_{i, k}^{\prime}=n_{i}$ for $i \neq k$. Similar to the definition of $\mathbf{b}_{\mathbf{p d}}$, the form of $\mathbf{b}_{\mathbf{s d}}^{\mathbf{k}}$ and its action space is defined as:

$$
\begin{aligned}
\mathbf{b}_{\mathbf{s d}}^{\mathbf{k}} & =\left\{\left(b_{s d}^{1, k}, b_{s d}^{2, k}, \ldots, b_{s d}^{K, k}\right): \sum_{i=1}^{K} b_{s d}^{i, k}=0,\right. \\
& -n_{i, k}^{\prime} \leq b_{s d}^{i, k} \leq\left\lfloor\frac{(M-m) C}{B_{i}}\right\rfloor-n_{i, k}^{\prime} \text { for } 1 \leq i \leq K, \\
& \sum_{i=1}^{K} B_{i}\left(n_{i, k}^{\prime}+b_{s d}^{i, k}\right) \leq(M-m) C, \\
& \text { if } \left.\exists j\left(1 \leq j \leq K, b_{s d}^{j, k}>0\right) \text { then } b_{s d}^{i, k} \geq 0 \text { for } \forall i>j\right\} .
\end{aligned}
$$

It is worth noting that the actions above do not need to consider how to adapt the bandwidth for each individual SU, thus leaving the flexibility to the algorithm design. More finetuned bandwidth allocation algorithms can be developed on top of the actions defined in this paper to achieve their specific objectives. For instance, the throughput fairness among SUs can be enforced by adopting an algorithm to select which SU to upgrade or degrade its QoS level in each round.

\section{State Transition Probability}

Let $\tau_{\mathbf{s}}(\mathbf{a})$ be the expected sojourn time in state $\mathbf{s}$ when action $\mathbf{a}$ is chosen. The exponential distribution of the interarrival and service time of the PUs and SUs yields:

$$
\tau_{\mathbf{s}}(\mathbf{a})=\left(a_{s} \lambda_{s}+a_{p} \lambda_{p}+\left(\sum_{i=1}^{K} n_{i}\right) \mu_{s}+m \mu_{p}\right)^{-1} .
$$

The probability that the system will transit to the state $\mathbf{s}^{\prime}=$ $\left(m^{\prime}, \mathbf{n}^{\prime}\right)$ at the next decision epoch given the current state of the system is $\mathbf{s}=(m, \mathbf{n})$ is:

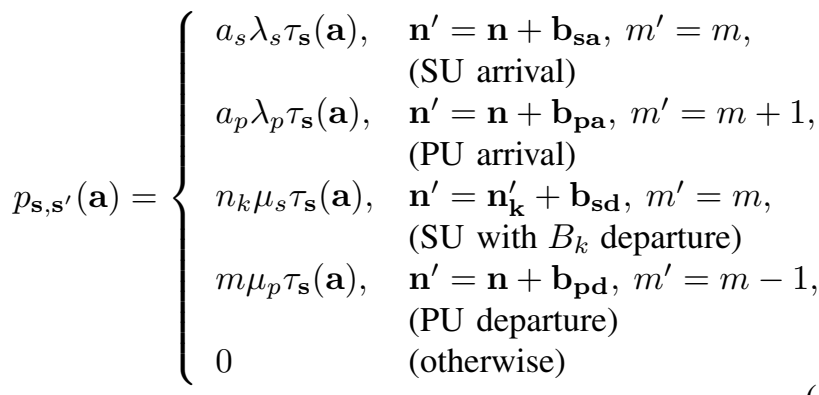

\section{Reward and Cost}

Different actions chosen in a state will result in different rewards and costs. On one hand, the reward earned by the network operator is generally proportional to the number of the admitted SUs and the bandwidths allocated to them. On the other hand, bandwidth adaptation operations require extra signalling overhead for service level and radio access level negotiation, incurring the non-trivial operational cost. Whereas the detailed definition of the cost function depends on the specific network environment, one general intuition is that the cost should be proportional to the number of bandwidth adaptation operations. Let $r_{\mathbf{s}}(\mathbf{a})$ and $c_{\mathbf{s}}(\mathbf{a})$ denote the earned reward and the incurred cost until the next decision epoch 
given action $a$ is taken at state $s$, respectively. $r_{\mathbf{s}}(\mathbf{a})$ is defined as:

$$
r_{\mathbf{s}}(\mathbf{a})=\sum_{i=1}^{K} B_{k} n_{k} \tau_{\mathbf{s}}(\mathbf{a})+\gamma a_{s} \lambda_{s} \tau_{\mathbf{s}}(\mathbf{a}),
$$

where the first term models the reward generated from the spectrum utilization over time and the second term models a one-time reward earned by admitting a new SU with an arrival probability $\lambda_{s} \tau_{\mathbf{s}}(\mathbf{a}) . \gamma$ is a weight factor. $c_{\mathbf{s}}(\mathbf{a})$ is given as:

$$
c_{\mathbf{s}}(\mathbf{a})=c_{b} N_{\mathbf{s}}(\mathbf{a}),
$$

where $c_{b}$ denotes the cost of one bandwidth adaptation operation and $N_{\mathbf{s}}(\mathbf{a})$ denotes the expected number of bandwidth adaptation operations when action $a$ is taken in state $s$, given by:

$$
\begin{aligned}
N_{\mathbf{s}}(\mathbf{a}) & =a_{s} \lambda_{s} \tau_{\mathbf{s}}(\mathbf{a}) \sum_{i=1}^{K} I\left(b_{s a}^{i}>0\right) b_{s a}^{i} \\
& +a_{p} \lambda_{p} \tau_{\mathbf{s}}(\mathbf{a}) \sum_{i=1}^{K} I\left(b_{p a}^{i}>0\right) b_{p a}^{i} \\
& +\sum_{k=1}^{K}\left(n_{k} \mu_{s} \tau_{\mathbf{s}}(\mathbf{a}) \sum_{i=1}^{K} I\left(b_{s d}^{i, k}>0\right) b_{s d}^{i, k}\right) \\
& +m \mu_{p} \tau_{\mathbf{s}}(\mathbf{a}) \sum_{i=1}^{K} I\left(b_{p d}^{i}>0\right) b_{p d}^{i},
\end{aligned}
$$

where $I(\cdot)$ is an indicator $(I(\cdot)=1$, if condition in $(\cdot)$ is satisfied; $I(\cdot)=0$, otherwise). Note that the cost of the eviction operations are not considered here but will be considered as a constraint in the optimization framework in the following section.

\section{Optimal STRATEGY FOR QOS PROVISION}

To find the optimal action for each possible system state to maximize the long-term network revenue as a function of the reward and cost while keeping the forced dropping probability of the SUs upper-bounded, we formulate a linear programming algorithm:

Maximize revenue:

$$
\sum_{\mathbf{s} \in \mathbf{S}} \sum_{\mathbf{a} \in \mathbf{A}(\mathbf{s})}\left(r_{\mathbf{s}}(\mathbf{a})-c_{\mathbf{s}}(\mathbf{a})\right) z_{\mathbf{s}, \mathbf{a}},
$$

Subject to:

$$
\begin{gathered}
\sum_{\mathbf{s} \in \mathbf{S}} \sum_{\mathbf{a} \in \mathbf{A}(\mathbf{s})} \tau_{\mathbf{s}}(\mathbf{a}) z_{\mathbf{s}, \mathbf{a}}=1, \\
\sum_{\mathbf{a} \in \mathbf{A}\left(\mathbf{s}^{\prime}\right)} z_{\mathbf{s}, \mathbf{a}}-\sum_{\mathbf{s} \in \mathbf{S}} \sum_{\mathbf{a} \in \mathbf{A}(\mathbf{s})} p_{\mathbf{s}, \mathbf{s}^{\prime}}(\mathbf{a}) z_{\mathbf{s}, \mathbf{a}}, \forall \mathbf{s}^{\prime} \in \mathbf{S}, \\
\sum_{\mathbf{s} \in \mathbf{S} \mathbf{a} \in \mathbf{A}(\mathbf{s})} \sum_{p} e a_{p} \lambda_{p} \tau_{\mathbf{s}}(\mathbf{a}) z_{\mathbf{s}, \mathbf{a}} \\
\leq P_{d}^{\text {Bound }} \sum_{\mathbf{s} \in \mathbf{S}} \sum_{\mathbf{a} \in \mathbf{A}(\mathbf{s})} a_{s} \lambda_{s} \tau_{\mathbf{s}}(\mathbf{a}) z_{\mathbf{s}, \mathbf{a}},
\end{gathered}
$$

where variable $z_{\mathbf{s}, \mathbf{a}} \geq 0$ can be explained as the long run fraction of the decision epochs at which the system is in state
TABLE I

EXPERIMENTAL PARAMETERS

\begin{tabular}{|c|c||c|c|}
\hline$M$ & 3 & $\lambda_{s}$ & 0.5 \\
\hline$C$ & 3 & $\mu_{s}$ & 0.5 \\
\hline$K$ & 2 & $\mu_{p}$ & 0.1 \\
\hline$B_{1}$ & 1 & $P_{d}^{\text {Bound }}$ & $1 \%$ \\
\hline$B_{2}$ & 2 & $\gamma$ & 1.0 \\
\hline
\end{tabular}

$s$ and action $a$ is chosen. Constraint (15) and constraint (16) represent the normalization and balance equation, respectively. The forced dropping probability of the SUs $P_{d}$ is expressed as:

$$
P_{d}=\frac{\sum_{\mathbf{s} \in \mathbf{S}} \sum_{\mathbf{a} \in \mathbf{A}(\mathbf{s})} e a_{p} \lambda_{p} \tau_{\mathbf{s}}(\mathbf{a}) z_{\mathbf{s}, \mathbf{a}}}{\sum_{\mathbf{s} \in \mathbf{S}} \sum_{\mathbf{a} \in \mathbf{A}(\mathbf{s})} a_{s} \lambda_{s} \tau_{\mathbf{s}}(\mathbf{a}) z_{\mathbf{s}, \mathbf{a}}},
$$

Enforcing an upper bound $P_{d}^{\text {Bound }}$ for $P_{d}$ yields (17).

\section{Performance Evaluation}

In this section, the performance of the proposed strategy based on the SMDP (referred as SMDP_aBA) is evaluated and compared with three other schemes: threshold-based channel reservation scheme without bandwidth adaptation (referred as Threshold_maxBA), threshold-based channel reservation scheme with bandwidth adaptation (referred as Threshold_aBA), and the optimal strategy based on the SMDP but without bandwidth adaptation (referred as SMDP_maxBA). In both schemes without bandwidth adaptation, the maximum bandwidth requirement of each SU needs to be satisfied all the time. The operation of Threshold_aBA is summarized as follows: when a new SU arrives, the existing SUs subsequently degrade their QoS levels to the next lower level to accommodate the new SU if needed and the new SU is rejected if all the SUs have degraded their QoS levels to the lowest level but the remaining spectrum bandwidth is still less than a threshold; when a PU arrives, if all the SUs have degraded their QoS levels to the lowest level but still cannot find enough spectrum bandwidth to support all of them. Portion or all of the SUs will be evicted subsequently until the remaining SUs can be supported. When a SU or a PU departs, the SUs will subsequently upgrade their QoS levels to the next higher level until approaching the threshold. For fair comparison, the number of bandwidth adaptation operations is counted only based on the initial QoS level and the final QoS level. The parameters fixed in evaluation are listed in Table I. The GLPK tool [10] is used to solve the linear program in this paper.

To fully exploit the benefit of adaptive bandwidth allocation, we first set the cost of one bandwidth adaptation operation $c_{b}=0$ and evaluate the average utilized spectrum and SU blocking probability with respect to varying PU arrival rates. For all the schemes, the forced dropping probability of the SUs is required to be below the pre-defined bound. For the threshold-based schemes, the results are derived when the optimal threshold is used for each PU arrival rate. As shown in Fig.1 and Fig.2, bandwidth adaptation can significantly improve the spectrum utilization and reduce the SU blocking 


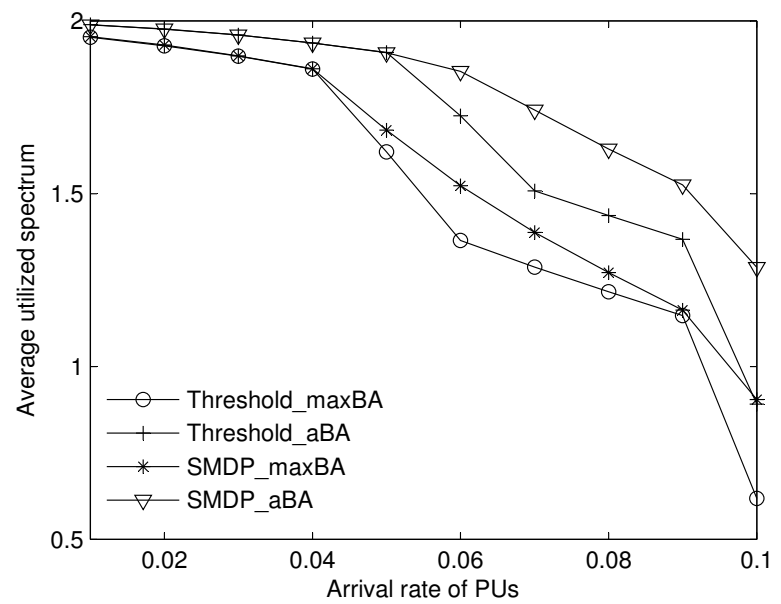

Fig. 1. Average utilized spectrum vs. arrival rate of PUs.

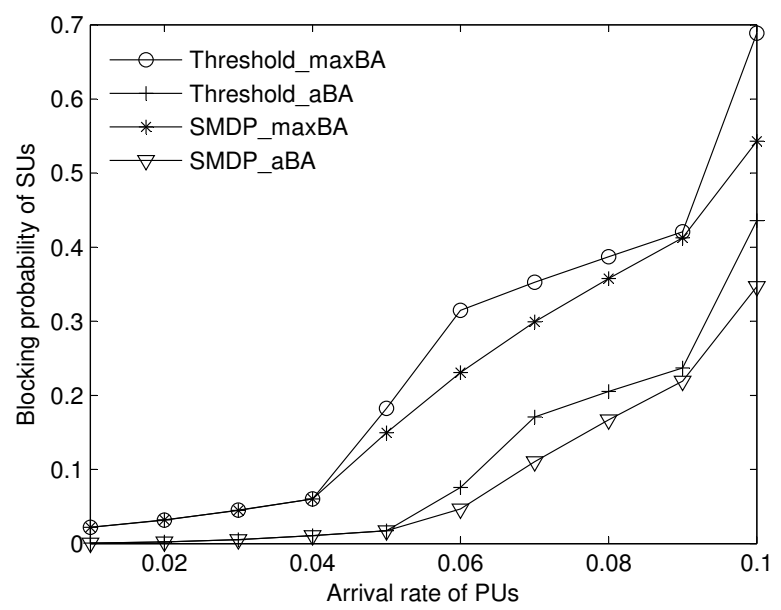

Fig. 2. Blocking probability of SUs vs. arrival rate of PUs.

probability. The SMDP-based schemes always outperform or at least equal to the corresponding threshold-based schemes with varying PU arrival rates. That is due to the fact that the threshold-based schemes cannot adapt the decision for each specific system state.

We then set the PU arrival rate $\lambda_{p}=0.05$ and evaluate the impact of the cost per bandwidth adaptation on the average network revenue, which is calculated from (14). As shown in Fig.3, the proposed scheme SMDP_aBA can adapt to the cost per bandwidth adaptation and decide whether or not a bandwidth adaptation is adopted with the objective to maximize the long-term network revenue. On the other side, Threshold_aBA cannot take the bandwidth adaptation cost into account. Its performance drops quickly with the increase of the cost and can be even worse than the schemes without bandwidth adaptation.

\section{CONCLUSION}

In this paper, we propose an optimal decision-making framework for joint admission control, eviction control and bandwidth adaptation to support the QoS of the SUs under spectrum mobility in cognitive radio networks. By formulating

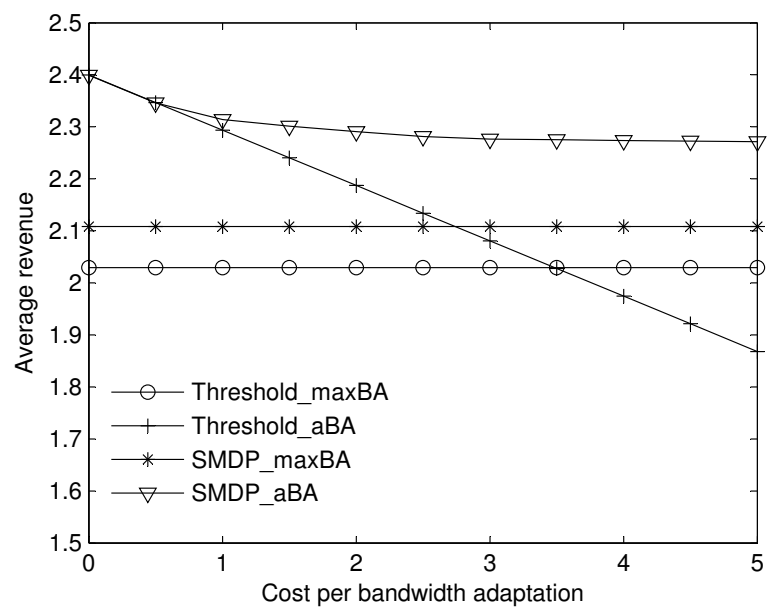

Fig. 3. Average revenue vs. cost per bandwidth adaptation.

the problem as a SMDP solved via a LP algorithm, the optimal decision at each system state is derived to maximize the long-term network revenue. Compared to the state-of-theart schemes, the proposed scheme is shown to improve the spectrum utilization and reduce the SU blocking probability while keeping the forced dropping probability of the SUs upper-bounded. Furthermore, it is shown that the proposed scheme can adapt to the bandwidth adaptation cost. For future work, we would like to extend the problem to jointly consider both physical mobility due to the SU movement and spectrum mobility due to the PU activity.

\section{ACKNOWLEDGMENT}

The research leading to these results was derived from the European Community's Seventh Framework Programme (FP7) under Grant Agreement number 248454 (QoSMOS) and 257626 (ACROPOLIS).

\section{REFERENCES}

[1] I. F. Akyildiz, W.-Y. Lee, M. C. Vuran, and S. Mohantly, "Next generation/dynamic spectrum access/cognitive radio wireless network: a survey," Computer Networks, vol. 50, no. 13, pp. 2127-2159, 2006.

[2] X. Zhu and L. Shen, "Analysis of cognitive radio spectrum access with optimal channel reservation," IEEE Commun. Lett., vol. 11, no. 4, pp. 304-306, 2007.

[3] D. Paramo, V. Pla, and J. Bauset, "Optimal admission control in cognitive radio networks," in Proc. IEEE CROWNCOM'09, 2009.

[4] M. Huang, R. Yu, and Y. Zhang, "Call admission control with soft-QoS based spectrum handoff in cognitive radio networks," in Proc. ACM IWCMC'09, 2009.

[5] "Advanced video coding for generic audio-visual services," ITU-T Recommendation H.264 and ISO/IEC 14496-10 AVC, 2003.

[6] H. Kim and K. G. Shin, "Optimal admission and eviction control of secondary users at cognitive radio hotspots," in Proc. IEEE SECON'09, 2009.

[7] Y. Xiao, P. Chen, and Y. Wang, "Optimal admission control for multiclass of wireless adaptive multimedia services," IEICE Trans. Commun., vol. E84-B, no. 4, pp. 795-804, 2001.

[8] F. Yu, V. W. S. Wong, and V. C. M. Leung, "A new QoS provisioning method for adaptive multimedia in cellular wireless networks," in Proc. IEEE INFOCOM'04, 2004.

[9] K. W. Ross and D. H. K. Tsang, "Optimal circuit access policies in an ISDN environment: A Markov decision approach," IEEE Trans. Commun., vol. 37, no. 9, pp. 934-939, 1989.

[10] GNU Linear Programming Kit: http://www.gnu.org/software/glpk/. 